\title{
Simulating Aqueous Adsorption of Polycyclic Aromatic Hydrocarbons on Graphene and Graphene Oxide Quantum Dots
}

\author{
Yiwen Liu ${ }^{\mathrm{a}}$, Zhuang Wang ${ }^{\mathrm{b} *}$, Se Wang ${ }^{\mathrm{c}}$ and Hao Fang ${ }^{\mathrm{d}}$ \\ Collaborative Innovation Center of Atmospheric Environment and Equipment Technology (AEET), \\ School of Environmental Science and Engineering, Nanjing University of Information Science and \\ Technology, Nanjing 210044, China \\ ayiwenliu20@163.com, b'zhuang.wang@nuist.edu.cn, cwangse@nuist.edu.cn, \\ dfang.hao@vip.163.com
}

${ }^{*}$ Correspondence should be addressed to Zhuang Wang: zhuang.wang@nuist.edu.cn

Keywords: Graphene; Graphene oxide; Polycyclic aromatic hydrocarbons; Molecular Simulation Abstract. Interactions of polycyclic aromatic hydrocarbons (PAHs) with graphene quantum dot (GQD) and graphene oxide quantum dot (GOQD) were probed by local density approximation of density functional theory with empirical dispersion correction. Simulation reveals that van der Waals force is the main mechanism for the GQD-PAH and GOQD-PAH interactions and the van der Waals interaction is mainly dispersion interaction. The computed adsorption energies of the GQD-PAH systems in the aqueous phase exhibited a positive linear correlation with the hydrophobicity of PAH molecules, while the GOQD-PAH systems showed a relatively weak correlation. This implies that hydrophobic interactions play an important role in the interactions between PAHs and GQD. Furthermore, the hydrophobic effect contributes more to the adsorption mechanism through increasing the number of the PAH rings.

\section{Introduction}

Graphene is a typical carbonaceous nanomaterial consisting of one monolayer of carbon atoms arranged in a two-dimensional honeycomb lattice [1]. Graphene exhibits many outstanding physical and chemical properties because of sp2-hybridized carbon atoms [2]. The unique properties of graphene (such as huge surface area, high stability, and strong adsorption ability) have paved the way to its potential capacity for environmental applications [3]. Graphene oxide, which is oxidized graphite with enhanced aqueous solubility, has also been applied in various fields, such as graphene manufacture, coating, flexible rechargeable battery electrode, and water purification [4].

It was previously revealed that Graphene and graphene oxide show excellent performance in adsorption with many organic pollutants [5], in particular for polycyclic aromatic hydrocarbons (PAHs) [6]. However, molecular mechanism for the adsorption of PAHs by graphene materials was still being debated. For instance, graphene displayed high affinity to PAHs by $\pi-\pi$ interactions to the flat surface, whereas graphene oxide adsorption was significantly reduced after oxygen-containing groups were attached to graphene surfaces [6]. In addition, the dispersive interaction is the most important contribution to the binding of PAH molecules onto graphene [7]. Therefore, more studies are needed to probe the potential adsorption behaviors of graphene materials at a molecular level.

The aim of the present study was to elucidate adsorption mechanism of PAHs by graphene materials. Local density approximation (LDA) with dispersion correction was carried out to estimate the equilibrium geometry, adsorption energy $\left(E_{\mathrm{a}}\right)$, and charge transfer.

\section{Computational Methods}

The studied system. Eight PAHs, i.e., naphthalene (NAP), fluorene (FLU), phenanthrene (PHE), anthracene (ANT), pyrene (PYR), fluoranthene (FLT), benzo[a]anthracene (BaA), and benzo[b]fluoranthene $(\mathrm{BbF})$, were selected for this study as adsorbates. The constructed adsorbents included coronene, a monolayer graphene quantum dot (GQD), and a monolayer graphene oxide 
quantum dot (GOQD). The GQD is composed of 70 carbon atoms and 22 saturated hydrogen atoms. The GOQD consists of 50 carbon atoms, 11 oxygen atoms, and 24 saturated hydrogen atoms.

Theoretical computation. Forcite Plus code was used to obtain the initial optimized structures of the studied systems at a molecular mechanics (MM) level. The atomic configuration with the lowest total energy for each system was built as a set of inputs for geometry optimization and properties calculation at a quantum mechanics (QM) level. In this study, the local density approximation (LDA) and the LDA with the DFT-correction (DFT-D method of OBS) methods were employed to calculate the isolated molecules and compounds. The conductor like screening model (COSMO) was employed to simulate the solvent (water) environment [8]. The dielectric constant for water was chosen to be 78.54. The obtained structures were optimized with the COSMO model using the LDA+DFT-D functional. The charge transfer between the adsorbate and adsorbent molecules was estimated by the Mulliken population analysis.

\section{Results and Discussion}

The computed $E_{\mathrm{a}}$ values derived from van der Waals energies $\left(E_{\mathrm{v}-\mathrm{a}}\right)$ between the PAHs and GQD/GOQD by the MM method are shown in Fig. 1, which are approximate to the $E_{\text {a }}$ values derived from the potential energies $\left(E_{\mathrm{p}-\mathrm{a}}\right)$. This indicates that van der Waals interaction mainly contributes to the interaction mechanisms for the GQD-PAHs and GOQD-PAHs interactions. Furthermore, the main bodies of both GQD and GOQD are composed of sp2 hybridized carbon atoms, implying that van der Waals interaction may contribute equally to the GQD-PAH and GOQD-PAH systems.
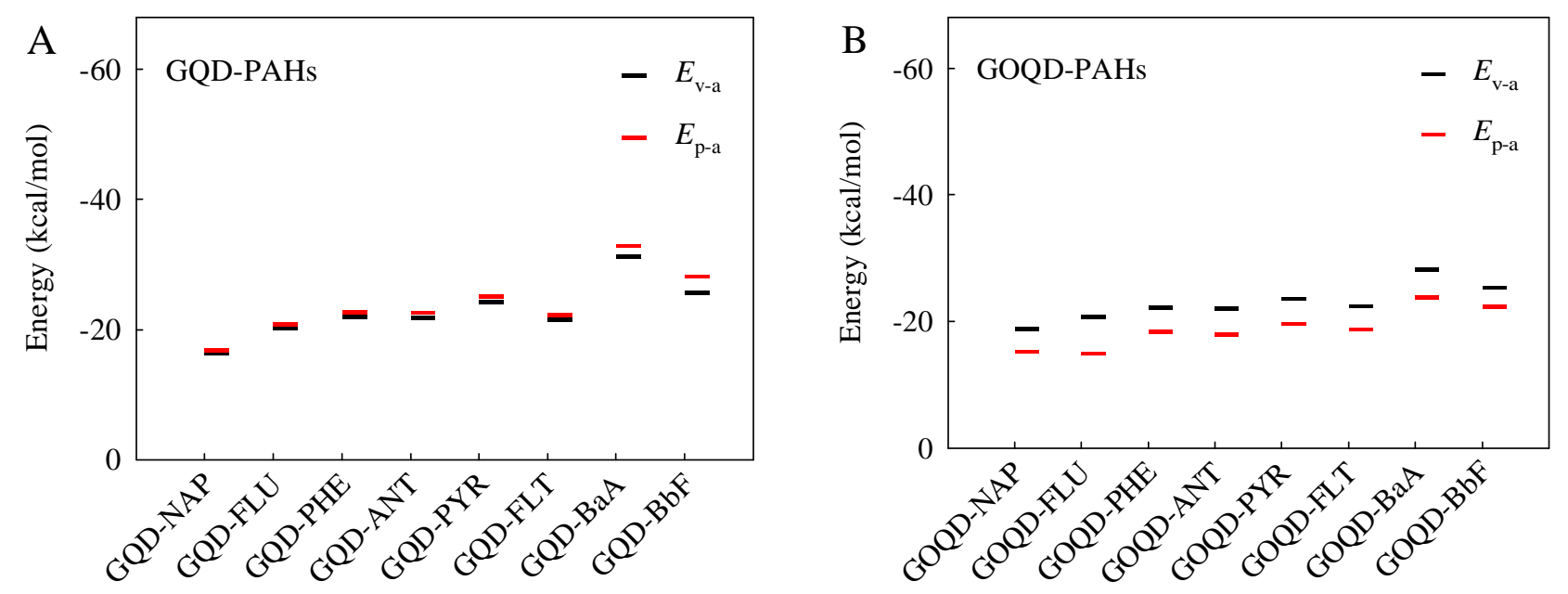

Fig. 1. Calculated potential energy adsorption energies $\left(E_{\mathrm{p}-\mathrm{a}}\right)$ and van der Waals adsorption energies $\left(E_{\mathrm{v}-\mathrm{a}}\right)$ for the PAHs molecules adsorbed upon the GQD (A) and GOQD (B) surfaces using the MM method

To precisely elucidate the interaction mechanisms, we have used the quantum-chemical parameters including the $E_{\mathrm{a}}$ values derived from the total energies $\left(E_{\mathrm{t}-\mathrm{a}}\right)$, molecule-adsorbent distances, and charge transfer (Table 1). The $E_{\mathrm{t}-\mathrm{a}}$ obtained at the LDA+DFT-D level are significantly higher than the adsorption energies from the LDA level. This suggests that the dispersion interactions were found to play the main role in the adsorption of PAHs on the graphene material surfaces. For the GQD-PAH systems, the absolute $E_{\mathrm{t}-\mathrm{a}}$ values obtained at the LDA+DFT-D level increase with the increase of the PAH ring numbers. However, for the GOQD-PAH systems, the PAH ring numbers have no impacts on the GOQD-PAH interactions. Generally, the absolute $E_{\mathrm{t}-\mathrm{a}}$ values of the PAHs on the GQD are higher than those of the PAHs on the GOQD, implying that the GQD exhibited higher adsorption capacity for the PAHs than the GOQD. An experimental study by Wang et al. [6] also implies that GQD displayed high affinity to PAHs, whereas GOQD adsorption was significantly reduced after oxygen-containing groups were attached to GQD surfaces. 
Table 1. Calculated parameters of the PAH adsorption systems including the adsorption distances $(d)$, the adsorption energy derived from total energies $\left(E_{\mathrm{t}-\mathrm{a}}\right)$, the charge transfer $(q)$ as well as the logarithm of the n-octanol/water partition coefficient $\left(\log K_{\text {OW }}\right)$ values of the PAH congeners

\begin{tabular}{|c|c|c|c|c|c|c|c|c|c|}
\hline \multirow{3}{*}{ PAHs } & \multirow{3}{*}{$\log K_{\mathrm{OW}}$} & \multicolumn{2}{|c|}{$d(\hat{\AA})^{c}$} & \multicolumn{4}{|c|}{$E_{\mathrm{t}-\mathrm{a}}(\mathrm{kcal} / \mathrm{mol})$} & \multicolumn{2}{|c|}{$\Delta q(\mathrm{e})^{d}$} \\
\hline & & \multirow{2}{*}{ GQD } & \multirow{2}{*}{ GOQD } & \multicolumn{2}{|l|}{ GQD } & \multicolumn{2}{|c|}{ GOQD } & \multirow{2}{*}{ GQD } & \multirow{2}{*}{ GOQD } \\
\hline & & & & LDA+DFT-D & LDA & LDA+DFT-D & LDA & & \\
\hline NAP & $3.36^{a}$ & 2.54 & 3.03 & -52.55 & -11.75 & -58.41 & -15.18 & -0.006 & 0.003 \\
\hline FLU & $4.18^{b}$ & 2.68 & 3.10 & -66.06 & -15.28 & -64.83 & -14.38 & -0.002 & 0.031 \\
\hline PHE & $4.57^{b}$ & 2.60 & 4.00 & -70.15 & -15.87 & -61.22 & -10.59 & -0.026 & 0.008 \\
\hline ANT & $4.54^{a}$ & 2.65 & 3.24 & -67.24 & -14.58 & -62.97 & -14.87 & -0.026 & 0.101 \\
\hline PYR & $5.18^{b}$ & 2.57 & 3.47 & -77.31 & -17.87 & -67.88 & -16.08 & -0.032 & 0.018 \\
\hline FLT & $5.22^{b}$ & 2.64 & 3.27 & -75.26 & -16.65 & -55.55 & -8.91 & -0.046 & 0.007 \\
\hline $\mathrm{BaA}$ & $5.91^{b}$ & 2.76 & 3.20 & -99.76 & -22.45 & -73.64 & -12.10 & -0.063 & 0.065 \\
\hline $\mathrm{BbF}$ & $5.80^{b}$ & 2.74 & 3.87 & -94.86 & -20.50 & -65.84 & -14.75 & -0.057 & 0.027 \\
\hline
\end{tabular}

${ }^{a} \log K_{\mathrm{OW}}$ values are from ref 9.

${ }^{b} \log K_{\text {OW }}$ values are from ref 10 .

${ }^{c} d$ is a distance $(\AA)$ from the center mass of each PAH molecule to the surface of GQD or GOQD and was determined from the results of the LDA+DFT-D calculations.

${ }^{d} \Delta q(\mathrm{e})$ is the charge transfer between the PAH molecules and the adsorbents (the negative values indicate that the GQD is a charge donor; the positive values indicate that the GOQD is a charge acceptor) and was estimated by the

LDA+DFT-D method.

As shown in Table 1, the molecule-adsorbent distances are mostly shorter in the case of GQD-PAHs when compared with those of GOQD-PAHs obtained at the level of theory LDA+DFT-D. This confirms that the PAH compounds bind more strongly with the GQD surface than the GOQD surface. Additionally, Mulliken analysis results showed that only a little charge transfer $(<0.08$ e) occurs between each PAH and the GQD/GOQD (Table 1). This also means that there exist weak donor-acceptor interactions between the PAHs and the GQD/GOQD.
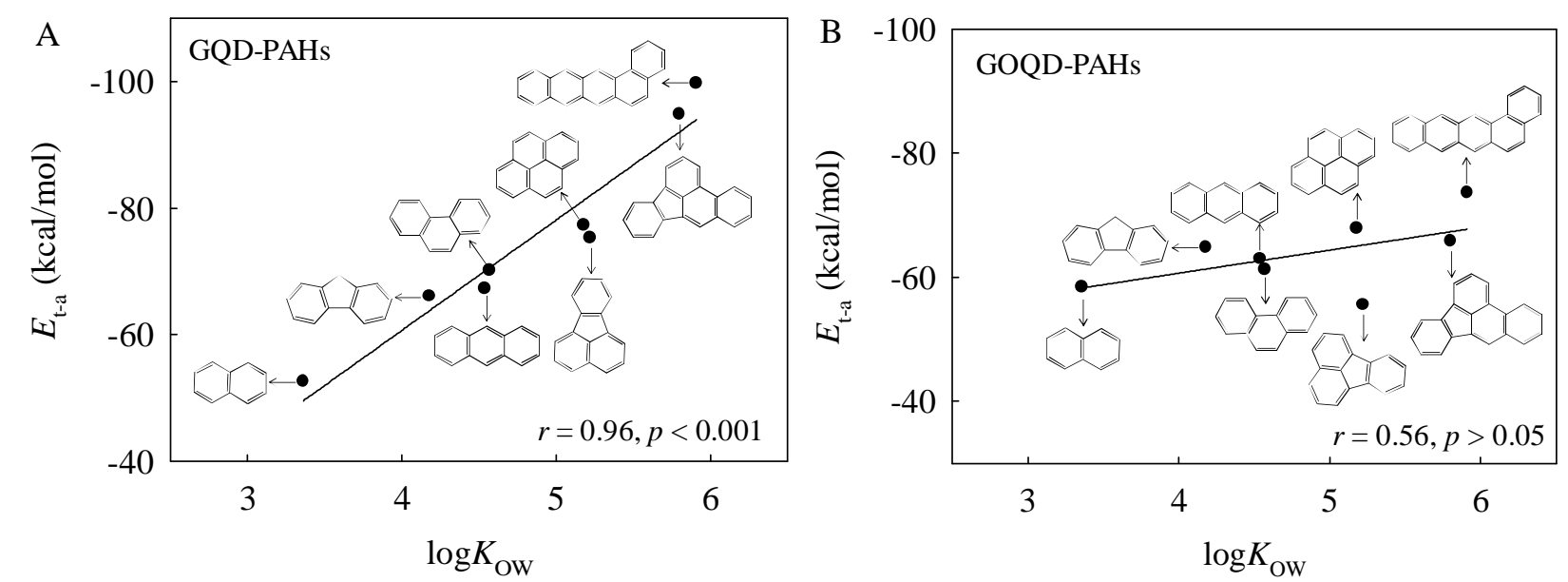

Fig. 2. Correlation between the $\log K_{\mathrm{OW}}$ values of PAHs and the adsorption energy values derived from the total energies $\left(E_{\mathrm{t}-\mathrm{a}}\right)$ of the GQD-PAH complexes $(\mathrm{A})$ and the GOQD-PAH complexes (B)

The polar functional groups on the edge of GOQD make it hydrophilic, while the hydrogen attached to the edge of GQD has no polarity, making the hydrophobic effect of the GQD-PAH system significant. Herein, to confirm the effect of hydrophobicity of PAHs on the adsorption strength, the relationship between the $E_{\mathrm{t}-\mathrm{a}}$ of the PAHs and the logarithm of the octanol-water partition coefficient $\left(\log K_{\mathrm{OW}}\right)$ of the PAHs was obtained (Fig. 2). For the GQD-PAH systems, the $E_{\mathrm{t}-\mathrm{a}}$ values correlate with $\log K_{\mathrm{OW}}$ significantly $(r=0.96, p<0.001)$ and increase with increasing $\log K_{\mathrm{OW}}$ (Fig. 2A). Thus, 
the stronger the hydrophobicity of the PAHs, the stronger their interactions with each GQD. As aforementioned, the absolute $E_{\mathrm{t}-\mathrm{a}}$ values for the GQD-PAH systems increase with the increase of the $\mathrm{PAH}$ ring numbers, implying that the hydrophobic effect contributes more to the adsorption mechanism through increasing the number of the PAH rings. However, for the GOQD-PAH systems, the $E_{\mathrm{t}-\mathrm{a}}$ values correlate with $\log K_{\mathrm{OW}}$ insignificantly $(r=0.56, p>0.05)$ (Fig. $\left.2 \mathrm{~B}\right)$. This may be due to the strong hydrophilicity and high polarity of GOQD. We can thus conclude that the interactions between GOQD and nonpolar PAH molecules are very weak compared with that of GQD.

\section{Summary}

We have found that the van der Waals force is the main mechanism for the GQD-PAH and GOQD-PAH interactions and the van der Waals interaction is mainly dispersion interaction. The computed $E_{\mathrm{a}}$ of the GQD-PAH systems in water exhibited a positive linear correlation with the hydrophobicity of the PAH molecules, while the GOQD-PAH systems showed a relatively weak correlation. The hydrophobic effect contributes more to the adsorption mechanism through increasing the number of the PAH rings.

\section{Acknowledgments}

We gratefully acknowledge the National Natural Science Foundation of China (Grant no. 21407080) and the Foundation Research Project of Jiangsu Province (nos. BK20140987 and BK20150891). We also thank the Startup Foundation for Introducing Talent (nos. 2013x044, 2014r020, and 2015r011), the open fund by Laboratory/Equipment Management Office (nos. $15 \mathrm{KF} 052$ and 15KF053), and the University Innovation Research/Training Program (nos. 201410300145 and 201510300106) of Nanjing University of Information Science and Technology.

\section{References}

[1] S. Stankovich, D. A. Dikin, G. H. Dommett, K. M. Kohlhaas, E. J. Zimney, E. A. Stach, R. D. Piner, S. T. Nguyen, R. S. Ruoff, Graphene-based composite materials, Nature. 442 (2006) 282-286. [2] M. J. Allen, V. C. Tung, R. B. Kaner, Honeycomb carbon: a review of graphene, Chemical Reviews. 110 (2010) 132-145.

[3] M. S. Mauter, M. Elimelech, Environmental applications of carbonbased nanomaterials, Environmental Science and Technology. 42 (2008) 843-5859.

[4] D. R. Dreyer, S. Park, C. W. Bielawski, R. S. Ruoff, The chemistry of graphene oxide, Chemical Society Reviews. 39 (2010) 228-240.

[5] N. Ding, X. Chen, C. M. Wu, Interactions between polybrominated diphenyl ethers and graphene surface: a DFT and MD investigation, Environmental Science: Nano. 1 (2014) 55-63.

[6] J. Wang, Z. Chen, B. Chen, Adsorption of polycyclic aromatic hydrocarbons by graphene and graphene oxide nanosheets, Environmental Science and Technology. 48 (2014) 4817-4825.

[7] O. V. Ershova, T. C. Lillestolen, E. Bichoutskaia, Study of polycyclic aromatic hydrocarbons adsorbed on graphene using density functional theory with empirical dispersion correction, Physical Chemistry Chemical Physics. 12 (2010) 6483-6491.

[8] A. Klamt, Conductor-like screening model for real solvents: a new approach to the quantitative calculation of salvation phenomena, Journal of Physical Chemistry. 99 (1995) 2224-2235.

[9] R. W. Walters, R. G. Luthy, Equilibrium adsorption of polycyclic aromatic hydrocarbons from water onto activated carbon, Environmental Science and Technology. 18 (1984) 395-403.

[10] D. Mackay, W. Y. Shiu, K. C. Ma, Illustrated handbook of physical-chemical properties and environmental fate for organic chemicals, Chelsea, MI, Vol. II, 1992. 